\title{
Photochemical bonding of epithelial cell-seeded collagen lattice to rat muscle layer for esophageal tissue engineering - a pilot study
}

\author{
${ }^{1}$ Chan BP, ${ }^{2}$ Sato M, ${ }^{3}$ Vacanti JP, ${ }^{4}$ Kochevar IE, ${ }^{4}$ Redmond RW \\ ${ }^{1}$ Medical Engineering, The University of Hong Kong, Pokfulam Road, \\ Hong Kong Special Administrative Region, China \\ ${ }^{2}$ Department of Surgery, Keio University School of Medicine, Tokyo, Japan \\ ${ }^{3}$ Tissue Engineering and Organ Fabrication Laboratory, Massachusetts General Hospital, \\ Boston, Massachusetts 02114 \\ ${ }^{4}$ Wellman Laboratories of Photomedicine, Department of Dermatology, \\ Massachusetts General Hospital, Boston, Massachusetts 02114 \\ Corresponding author: \\ BP Chan Ph.D. (bpchan@hkucc.hku.hk) \\ Research Assistant Professor, Medical Engineering Program, The University of Hong Kong, \\ Pokfulam Road, Hong Kong Special Administrative Region, China. \\ Tel.: (852) 2857 8553, Fax: (852) 28585415
}

\begin{abstract}
Bilayered tube structures consist of epithelial cell-seeded collagen lattice and muscle layer have been fabricated for esophageal tissue engineering. Good adhesion between layers in order to facilitate cell infiltration and neovascularization in the collagen lattice is required. Previous efforts include using other bioglues such as fibrin glue and silicone tube as the physical support. However, the former is subjected to chances of transmitting blood-born infectious disease and is time consuming while the latter requires a second surgical procedure. The current project aimed to bond the cell-seeded collagen lattice to muscle layer using photochemical bonding, which has previously been demonstrated a rapid and non-thermal procedure in bonding collagenous tissues.
\end{abstract}

Rat esophageal epithelial cells were seeded on collagen lattice and together with the latissimus dorsi muscle layer, were exposed to a photosensitizer rose Bengal at the bonding surface. An argon laser was used to irradiate the approximated layers. Bonding strength was measured during the peeling test of the collagen layer from the muscle layer. Post-bonding cell viability was assessed using a modified NADH-diaphorase microassay. A pilot in vivo study was conducted by directly bonding the cell-seeded collagen layer onto the muscle flap in rats and the structures were characterized histologically.

Photochemical bonding was found to significantly increase the adherence at the bonding interface without compromising the cell viability. This indicates the feasibility of using the technique to fabricate multi-layered structures in the presence of living cells. The pilot animal study demonstrated integration of the collagen lattice with the muscle layer at the bonding interface although the subsequent surgical manipulation disturbed the integration at some region. This means that an additional procedure removing the tube could be avoided if the approximation and thus the bonding are optimized. Cell infiltration and neovascularization were also evident demonstrating that direct bonding of engineered tissue structures in particular those with low processability such as collagen lattice to the host tissue is feasible.

\section{Keywords:}

Photochemical tissue bonding, artificial esophagus, epithelial cell-seeded collagen gel, muscle flap 


\section{Introduction}

In tissue engineering, fabrication of multi-layered tissue structures is a common approach. Lamination of thin layered structures with low processability such as those made of collagen gel is particularly difficult. This is because traditional mechanical devices such as sutures and staples would damage the delicate structures but do not give good integration. Other methods for lamination such as chemical ones using fibrin glues (Canonico 2003, Chao \& Torchiana 2003) and physical ones using radiations (Bass \& Treat 1995) are the potential alternatives. Nevertheless, the former usually takes a longer time for preparation and is subjected to the concerns relating to transmission of blood-born infectious diseases (Dunn \& Goa 1999) while the latter uses thermal coagulation of proteins as the bonding mechanism that is not suitable for engineered tissue structures where living cells are present.

Photochemical tissue bonding is an emerging laser-based non-thermal technique able to bond collagenous tissue structures such as skin grafts and tendon strips without compromising cell viability (Chan et al. 2002, 2004). The underlying mechanisms of the technique are not completely understood but are believed to associate with that of the photosensitization in direct and indirect pathways. In the direct pathway, the light activated photosensitizers interact with the surrounding molecules by direct hydrogen abstraction and this also produces reactive species such as free radicals and hydrogen peroxides, which are also reactive (Webster et al. 1989). In the indirect pathway, the light activated photosensitizers react with ground state oxygen molecules to produce active singlet oxygen, which diffuse to react with oxidizable groups in the surroundings (Balasubramanian et al. 1990). This photochemical bonding technique may present a simple, rapid and safe procedure in bonding layers of engineered tissue structures.

Artificial esophageal tissue replacements are to be anatomized to the defect after esophagectomy in cancer treatment. The design of the artificial esophagus is based on the normal histology of the esophageal tissue that an epithelial cell layer is seeding on a collagen lattice simulating the mucosal layer and then transplanted onto a muscle layer (Sato et al. 1993). The bilayered structure will be sealed to make tube-like structure for anastomosis. Previous approach was to use a silicone tube as the physical support, which compresses the cell-seeded collagen lattice against the muscle layer for one week before harvesting the structures for anastomisis (Yamamoto et al. 1999). However, this method has two important drawbacks, firstly, compressive force of the silicone tube may damage the epithelial cells and adversely affect the epithelialization process; secondly, a second surgical procedure is needed to remove the non-biodegradable silicone tube before anastomosis.

This pilot study aims to photochemically bond an engineered epithelial cell-seeded collagen lattice onto a muscle layer in order to make bilayered structure for esophageal tissue engineering. The adhesive force of the bonded structures and cell viability after bonding will be assessed ex vivo while a pilot animal model will be used to evaluate the bonding effect histologically.

\section{Materials and Methods}

\section{Epithelial cell seeded collagen lattice}

Epithelial cells were isolated from rat esophageal tissue by methods modified from that previously described (Sato et al. 1993). In brief, normal esophageal mucosa was digested in dispase for 3 hours at $37^{\circ} \mathrm{C}$ and the epithelial cells were released by incubating the epithelium with $0.025 \%$ Trypsin with $0.02 \%$ EDTA for 15 minutes at $37^{\circ} \mathrm{C}$. The epithelial cells were cultured for two passages in keratinocyte growth medium. Cells in the early passages at a concentration of $5 \times 10^{4}$ cells $/ \mathrm{ml}$ were seeded onto a piece of collagen lattice made 
from acid soluble collagen solution at a concentration of $2 \mathrm{mg} / \mathrm{ml}$. The cell seeded collagen lattice was cultured for 2 more weeks before bonding to muscle layers.

\section{$\underline{\text { Photochemical bonding of the cell-seeded collagen lattice onto muscle layer }}$}

Latissimus dorsi muscle layers from Sprague Dawley (SD) rats were dissected under dissection microscope and were carved into $1 \mathrm{~cm} \times 1 \mathrm{~cm}$ pieces using a surgical blade. The muscle layer was then secured onto a custom-made platform using 5-0 sutures. The cell-seeded collagen layer was punched into 6mm diameter discs under the dissection microscope and a small suture was attached to the collagen disc at the edge. The bottom side of the cell-seeded collagen layer and the upper side of the secured muscle layer was then stained with a photosensitizer, rose Bengal, at a concentration of $0.1 \%(\mathrm{w} / \mathrm{v})$ for 2 minutes. The collagen layer was then approximated to the muscle layer gently. An argon laser (Innova 100, Coherent, Palo Alto, CA), transmitted through a $1 \mathrm{~mm}$-diameter quartz fiber, was used to irradiate the approximated collagen latticemuscle bilayered composite at a spot size of $6 \mathrm{~mm}$ diameter. Specimens were irradiated at $0.5 \mathrm{~W} / \mathrm{cm}^{2}$ for 100 and 200 seconds prior to the measurement of the adherence force by peeling test as described previously (Chan et al. 2002). The suture loop was mounted onto a tensiometer (TCD-200, Chartillon), which is connected to a force gauge (DFA2, Ametek). A uniaxial force at a constant strain rate of $12.7 \mathrm{~mm} / \mathrm{min}$ was applied until the whole collagen lattice was peeled off from the muscle layer. The force-displacement curve was obtained and the adherence was calculated as the total area under the curve of the force-displacement curve $\left(\mathrm{mJ} / \mathrm{cm}^{2}\right)$.

\section{Cell viability assay after photochemical crosslinking}

Rat esophageal epithelial cells at a concentration of $5 \times 10^{4}$ cells $/ \mathrm{ml}$ were seeded on a piece of collagen lattice in each well of a 24 well culture plate and were cultured in the same medium for one week until confluence. An aliquot of $100 \mu \mathrm{l}$ of $0.1 \%$ rose Bengal was added into each well at the bottom of the culture plate for 2 minutes to stain the collagen gel after aspiration of excessive medium. The cell-seeded collagen gel was then irradiated with the same argon laser at an irradiance of $0.5 \mathrm{~W} / \mathrm{cm}^{2}$ for 100 and 200 seconds. The irradiated structure was incubated in the same medium for 2 hours in a $37^{\circ} \mathrm{C}$ incubator. The epithelial cell layer was then trypsinized using for a few minutes and was removed from the collagen lattice. The cell pellet was washed in medium with $10 \%$ fetal calf serum to stop the enzyme activity and then incubated with nicotinamide adenine dinucleotide (NADH)-diaphorase incubation fluid for 30 minutes at $37^{\circ} \mathrm{C}$. The NADHdiaphorase activity of viable cells would metabolize the substrates in the incubation fluid to form a water insoluble dark blue precipitate diformazan, which has an absorption peak at OD550 (Neumann et al. 1991). After thorough rinse, the cell pellet was lyzed and dissolved by $0.5 \mathrm{ml}$ DMSO for OD550 measurement using diformazan as standards.

\section{Pilot implantation of photochemically bonded structures}

Male SD rats were anaesthetized and a long incision was made at the back to expose the latissimus dorsi muscle. A muscle flap measured $2 \times 2 \mathrm{~cm}$ was made and set aside. After staining the bottom of the epithelial cell-seeded collagen lattice of the same size with $0.1 \%$ of rose Bengal solution for 2 minutes, the collagen gel was approximated to the muscle flap gently and irradiated with the same argon laser at a fluence of $100 \mathrm{~J} / \mathrm{cm}^{2}$. The bilayered structure was then made into a closed tube-like structure by sealing the sides and the two ends with 5-0 sutures. On the contralateral side, the collagen lattice was laid down onto the muscle flap and wrapped around a silicone tube. The side and the two ends were also sealed with sutures. The wound was closed and the animal was kept for 1 week before sacrifice. Upon sacrifice, the photochemically bonded structure and the contralacteral control tubes were exposed, and then opened by removing the sutures. The tissues were fixed in $10 \%$ buffered formalin overnight and five micron-thick sections were made after paraffin 
embedding. Routine histology was used to reveal the morphology of the bilayered structures to study the integration at the bonding interface.

\section{$\underline{\text { Statistics and data analysis }}$}

Data of all outcome measures were continuous in nature and verified for normal distribution so that parametric tests could be used. One-way ANOVA with Bonferroni's post-hoc tests were used to compare the adherence of the collagen lattice to the muscle layers and the cell viability in different treatment groups including the untreated control, the dye control and the laser control. SPSS 12.0 was used to execute the data analysis and the significance level was set at 0.05 .

\section{Results}

\section{Epithelial cell-seeded collagen lattice}

Fig. 1 showed the haematoxylin staining of the single layer human epithelial cell-seeded collagen lattice. The collagen lattice could be identified by the longitudinally running network to which a single layer of epithelial cells were seeded.

\section{$\underline{\text { Adherence of cell-seeded collagen lattice on muscle layer }}$}

Fig. 2 showed the box plot of the adherence of the cell-seeded collagen lattice to the muscle layer in different treatment groups. For the untreated control, dye and laser controls, there were only minimal adherence while for the photochemically bonded collagen lattice, there was at least 3 fold increase in adherence in groups with different fluences, 50 and $100 \mathrm{~J} / \mathrm{cm}^{2}$. One-way ANOVA with Bonferroni's post-hoc test showed that there was statistically significant difference among different treatment groups $(\mathrm{p}=0.002)$ and the photochemically bonded group $\left(50 \mathrm{~J} / \mathrm{cm}^{2}\right)$ showed significant difference comparing to all control groups $(\mathrm{p}<=0.011)$.

\section{Measurement of cell viability after photochemical bonding}

Fig. 3 showed the concentration of diformazan in viable epithelial cells in different treatment groups. It was demonstrated that the cell viability of all groups including the control groups and the photochemically bonded collagen lattice were similar. One-way ANOVA showed that there was no significant difference at all between the photochemically bonded group and other control groups $(\mathrm{p}=0.808)$.

\section{Implantation of cell-seeded collagen lattice bonded to muscle flap}

Fig. 4 showed the tube-like structures with the muscle flap on the outer side and the cell-seeded collagen lattice at the luminal side in SD rat. The diameter of the photochemically bonded tube-like structure (right) was smaller than that of the one using the silicone tube (left).

The luminal surface of the photochemically bonded bilayered structure was less smooth than that of the one using silicone tube because the rigid silicone tube was compressing against the cell-seeded collagen lattice on top of the muscle flap that resulting in a smooth luminal surface (Fig. 5).

H\&E staining of the cross-sectional morphology of the photochemically bonded bilayered structure (Fig. 6) showed that there was good integration between the cell-seeded collagen lattice and the muscle flap at the bonding interface that lot of cells, originated from the muscle layer, have been infiltrated into the collagen gel. Neovascularization was also evident near the bonding interface that small vessels were found. The epithelial 
cell layer at the top of the collagen layer was found at some region. The cell-seeded collagen lattice in the control group was much thinner than that of the photochemically bonded ones while cell infiltration and neovascularization were also evident. Both groups did not show further stratification at the epithelium.

\section{Discussion}

The current study demonstrated the feasibility of using a photochemical bonding technique to laminate cellseeded collagen lattice onto tissue layers that is important in fabricating multi-layer structures in tissue engineering. Ex vivo evidence showed enhanced adherence of collagen lattice on muscle layers (Fig. 2). In vivo pilot implantation study showed that photochemically bonded collagen lattice was able to adhere to the muscle flap to form the bilayered structure without detachment while integration at the bonding interface as shown by cell infiltration and neovascularization (Fig. 6). This showed that photochemical bonding may eliminate the use of the silicone tube and therefore avoids an additional surgical procedure in making artificial esophagus. This also prevents chronic compressive damage of the epithelial cells induced by the rigid silicone tube and therefore may result in a better epithelialization, which is the key to the success of functional replacement of esophagus.

Photochemical bonding also presents a safe procedure because the epithelial cells in the photochemically bonded groups remained as viable as the controls (Fig. 3). Potential cell damage in photochemical reactions is of two-fold. Firstly, thermal damage will be resulted if the heat accumulates due to high power and irradiance. Secondly, photochemical damage due to the cellular contact of the reactive oxygen species after photoactivation of the photosensitizing reagents such as free radicals and hydrogen peroxides. Nevertheless, both were absent in the current procedure because firstly the laser power used was safe according to our previous study (Chan et al. 2002) that the maximal temperature involved was below $40^{\circ} \mathrm{C}$ which does not induce thermal damage and secondly the photosensitizing reagent was only in contact with the lower side of the collagen lattice while the epithelial cells were seeded on top of the collagen lattice. As a result, the epithelial cells remained viable after the bonding procedure.

Epithelialization into multi-stratified epithelium with 20 layers like in human is important for esophageal functions (Miki et al. 1999). However, the current pilot study did not show further stratification of the epithelium. This may be due to the reason that the time of harvest was only one week post-implantation that is not sufficient for complete epithelialization. Further, the current pilot study did not incorporate keratinocyte growth factor or fibroblasts, which are known to secrete keratinocyte growth factors (Miki et al. 1999) that stimulate the epithelium growth. Future study using fibroblast as a feeder layer seeded within the collagen lattice should be conducted in order to optimize the conditions for growth of the epithelial cells.

Since photochemical tissue bonding requires proximal contact at molecular level between structures for bonding, good approximation between the collagen lattice and the muscle flap is necessary. Nevertheless, the approximated layers were easily disturbed during surgical manipulation such as sealing the layers into tubelike structures using sutures. This may explain why there is region without good integration in the histological pictures. In future studies, approximation between the collagen lattice and the muscle layer can be made after sealing the bilayered structures into tube-like structures by methods such as inserting an inflatable and non-adherent balloon into the bilayered tubes and removing after irradiation.

Last but not the least, many factors affecting the bonding efficiency such as the concentration of the photosensitizer and the light source parameters need to be optimized for best bonding results in future studies. 


\section{Conclusion}

The current pilot study has demonstrated the feasibility of using a photochemical bonding technique to laminate epithelial cell-seeded collagen lattice onto muscle layers with good adherence and integration without compromising cell viability in esophageal tissue engineering. The long term significance of the study is the development of safe technologies in fabricating multi-layered tissue engineered structures with living components.

\section{Acknowledgement}

The authors are grateful to Dr. Rox Anderson and Mr. Bill Faranelli for advice on laser optics and Dr. Beatrice Aveline for advice on rose Bengal photochemistry.

\section{References}

1. Balasubramanian D, Du X, Zigler JS Jr. (1990) The reaction of singlet oxygen with proteins, with special reference to crystallins. Photochem Photobiol. 52(4):761-8.

2. Bass LS, Treat MR. (1995) Laser tissue welding: a comprehensive review of current and future clinical applications. Lasers Surg Med. 17(4):315-49.

3. Canonico S. (2003) The use of human fibrin glue in the surgical operations. Acta Biomed Ateneo Parmense. 74 Suppl 2:21-5.

4. Chan BP et al. (2004) A light-activated tissue bonding in tendon repair - ex vivo and in vivo study. (J Surg Res, in print)

5. Chan BP, Kochevar IE, Redmond RW. (2002) Enhancement of porcine skin graft adherence using a light-activated process. J Surg Res.108(1):77-84.

6. Chao HH, Torchiana DF. (2003) BioGlue: albumin/glutaraldehyde sealant in cardiac surgery. Card Surg. 18(6):500-3.

7. Miki H, Ando N, Ozawa S, Sato M, Hayashi K, Kitajima M. (1999) An artificial esophagus constructed of cultured human esophageal epithelial cells, fibroblasts, polyglycolic acid mesh, and collagen. ASAIO J. 45(5):502-8.

8. Neumann RA, Knobler RM, Pieczkowski F, Gebhart W. (1991) Enzyme histochemical analysis of cell viability after argon laser-induced coagulation necrosis of the skin. J Am Acad Dermatol. 25(6 Pt 1):9918.

9. Sato M, Ando N, Ozawa S, Nagashima A, Kitajima M. (1993) A hybrid artificial esophagus using cultured human esophageal epithelial cells. ASAIO J. 39(3):M554-7.

10. Webster A, Britton D, Apap-Bologna A, Kemp G. (1989) A dye-photosensitized reaction that generates stable protein-protein crosslinks. Anal Biochem. 179(1):154-7.

11. Yamamoto Y, Nakamura T, Shimizu Y, Takimoto Y, Matsumoto K, Kiyotani T, Yu L, Ueda H, Sekine T, Tamura N. (1999) Experimental replacement of the thoracic esophagus with a bioabsorbable collagen sponge scaffold supported by a silicone stent in dogs. ASAIO J. 45(4):311-6. 




Fig. 1 - Single layer human epithelial cell-seeded collagen lattice (10X)

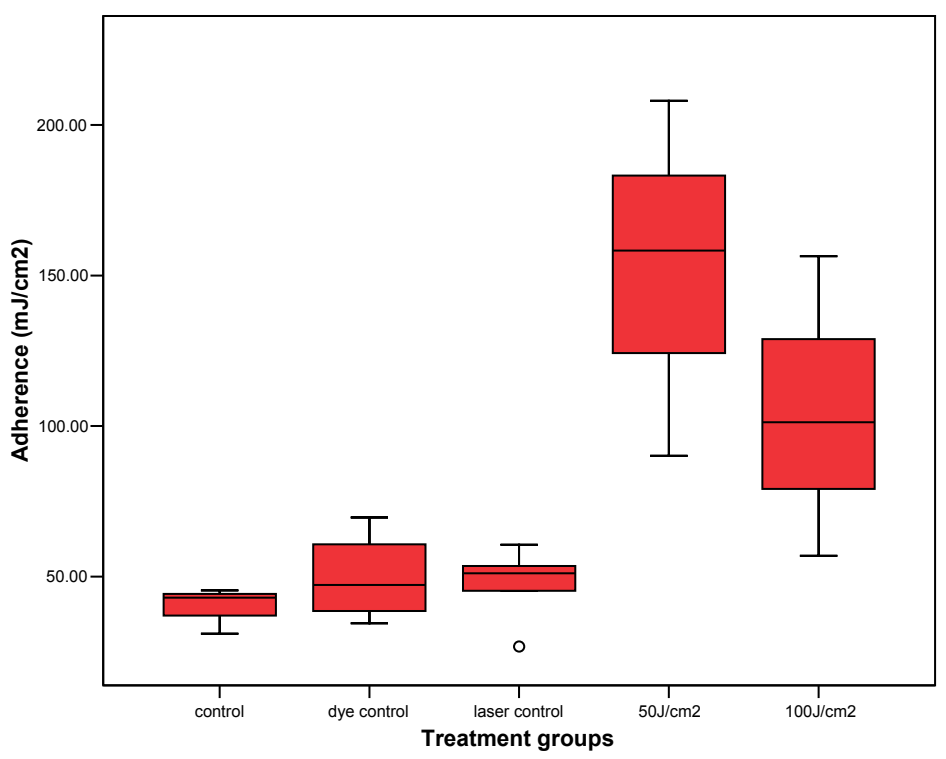

Fig. 2 - Box plot of adherence of cell-seeded collagen lattice on muscle layer in different treatment groups 


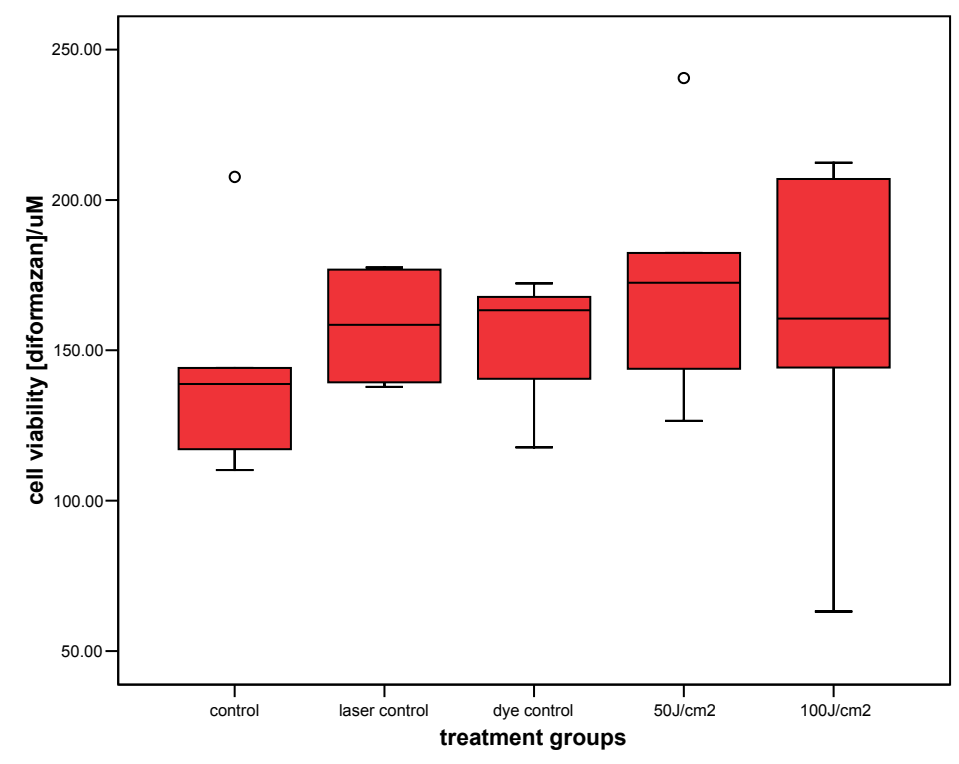

Fig. 3 - Box plot of cell viability in different treatment groups

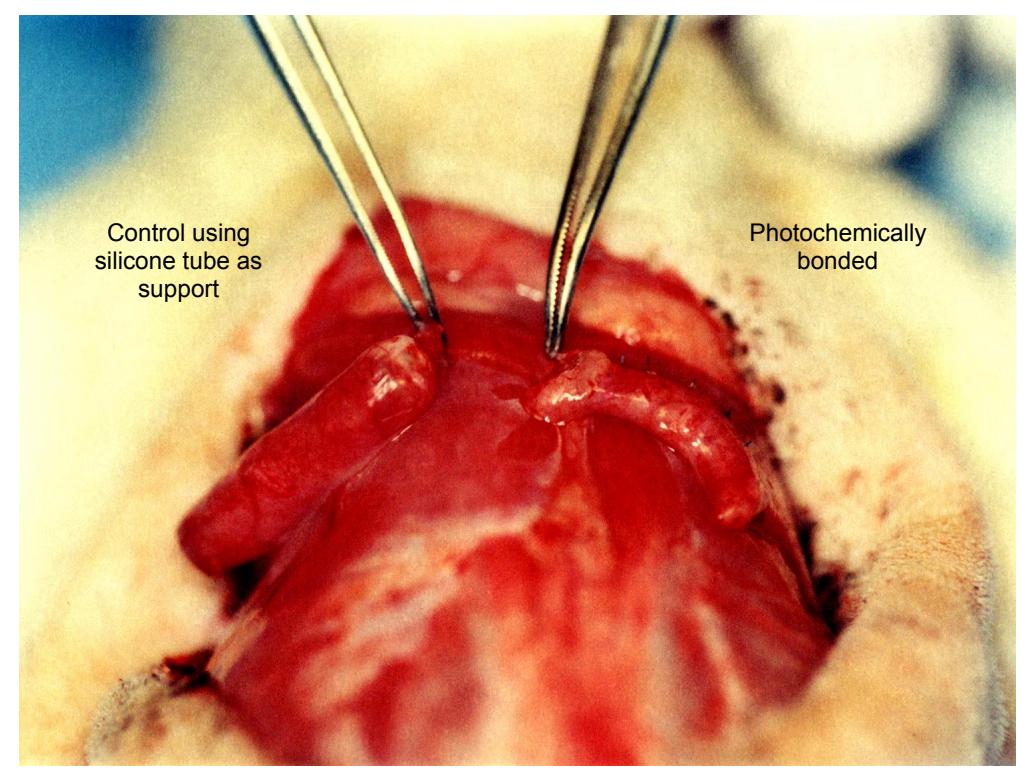

Fig. 4 - Formation of bilayered tube-like structures at the back of the SD rat 


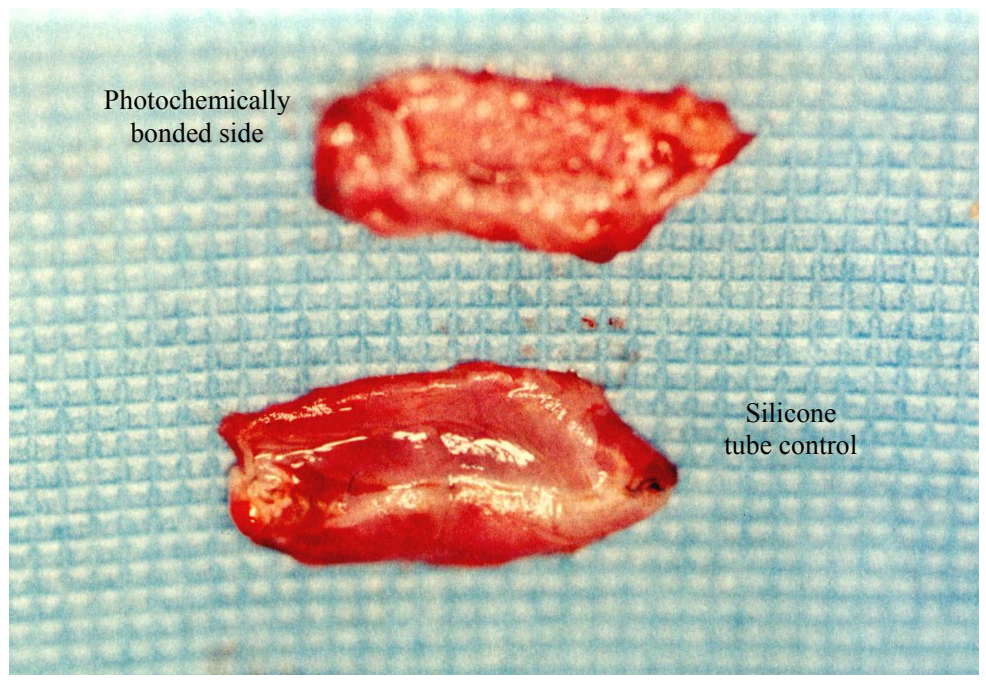

Fig. 5 - Interior of the tube-like structures
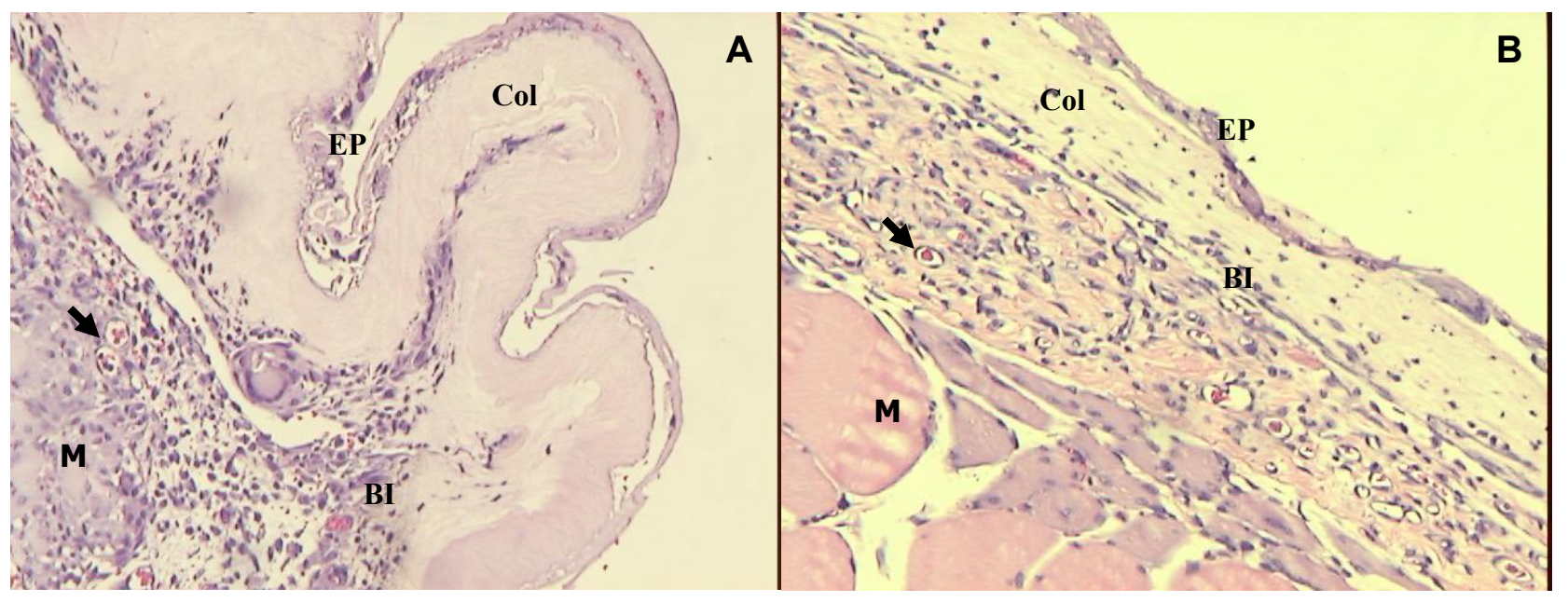

Fig. 6 - Routine $\mathrm{H} \& \mathrm{E}$ staining of photochemically bonded epithelial cell-seeded collagen lattice on muscle flap (A) and control side with silicone tube as physical support (B) (Magnification: 10X) $\mathrm{Col}$ - collagen lattice; EP - epithelial cell layer; $\mathrm{M}$ - muscle layer; BI - bonding interface; Arrow neovascularization. 